\title{
The proper timing of second-stage revision in treating periprosthetic knee infection: reliable indicators and risk factors
}

\author{
Jun $\mathrm{Fu}^{1+}$, Ming $\mathrm{Ni}^{1 \dagger}$, Heng $\mathrm{Li}^{2 \dagger}$, Xiang $\mathrm{Li}^{1}$, Wei Chai ${ }^{1}$, Yonggang Zhou ${ }^{1}$, Libo Hao ${ }^{1}$ and Jiying Chen ${ }^{*}$
}

\begin{abstract}
Background: Two-stage revision is the standard procedure for periprosthetic knee infection. But when to perform the second-stage is still under debate. We attempt to search the reliable indicators, risk factors, and proper timing for the second-stage revision.
\end{abstract}

Methods: We reviewed and followed 81 infected total knee arthroplasty patients who underwent two-stage revision from January 2010 to January 2014. Our cohort included 56 males and 25 females, all patients were confirmed as PJ with the same phenotypic cultures. The average age was $64.8 \pm 8.21$ (range 36-78) months. The mean follow-up time was $46.5 \pm 17.6$ (range 12-72) months after the second-stage surgeries. The diagnostic parameters including serum Creaction protein, erythrocyte sedimentation rate, and intraoperative frozen section at the time of re-implantation were analyzed. The spacer detention time and antibiotic treatment time were compared.

Results: Ten of them went through failed first- or second-stage surgeries. The overall success rate was $87.7 \%$. The intraoperative frozen section is a good indicator at the time of re-implantation; the sensitivity and specificity is 90 and 83.1\%. Serum CRP and ESR showed poor diagnostic value at time of re-implantation. Atypical pathogen infection, positive FS, and previous sinus were high-risk factors for failure of two-stage revision. Spacer detention time between 12 and 16 weeks had higher success rate than over 16 weeks.

Conclusion: The proper timing of re-implantation should be combined with disappearance of clinical symptoms and negative intraoperative FS with spacer detention time at 12 to 16 weeks.

Keywords: Periprosthetic joint infection, Total knee revision, Two-stage revision, Serum CRP, ESR, Intraoperative frozen section

\section{Background}

Periprosthetic joint infection (PJI) is the most devastated complication after total knee arthroplasties. The treatment option of PJI involves one- and two-stage revision. The standard procedure of one-stage revision includes the removal of infected prosthesis, thorough debridement and exchange of a new prosthesis. The two-stage revision requires antibiotic-loaded cement spacer implantation in the first stage and intravenous antibiotic treatment before the second stage. During the last two decades, both of the two protocols were reported with

\footnotetext{
* Correspondence: hy76yuy@163.com

†Jun Fu, Ming Ni and Heng Li contributed equally to this work.

${ }^{1}$ Department of Orthopaedics, Chinese People's Liberation Army General

Hospital (301 Hospital), Beijing 100853, China

Full list of author information is available at the end of the article
}

satisfied infection eradication rates. However, which treatment is more appropriate for patients depends on their clinical status, culture results, general health conditions, and even financial situations.

All the therapeutic efforts made before would be all in vain with the catastrophic recurrence of PJI. Sometimes failure is hard to avoid. The success rates of the two-stage revision vary from 4 to $41 \%$ [1]. This discrepancy of the reinfection rates could be attributed to inconsistent study factors including patient demographic characteristics, pathogenic characteristics, and different treating protocols. Moreover, no reliable reference standard for re-implantation is another cause. Therefore, how to rule out PJI at the time of second-stage revision is the key to a successful two-stage revision. 
So far, there is no authentic method to evaluate the control of PJI at the time of second-stage surgery. And there is also no clinical trial aiming at the best timing of second stage. Usually, surgeons had to make decisions basing on clinical symptoms combined with laboratory parameters. While in some cases, they can only rely on their own experiences. But, it is reported serologic markers such as C-reaction protein (CRP) and erythrocyte sedimentation (ESR) are not reliable markers at the time of second-stage surgery $[2,3]$. Although these laboratory indicators do decrease after intravenous antibiotic treatment, it is still difficult to rule out persist PJI. Elie reported both CRP and ESR poorly predicted the persistent infection and the cut-off values were difficult to obtain [3]. Some authors suggested using the Musculoskeletal Infection Society (MSIS) criteria to assess infection at the time of second stage. It has been reported that both MSIS criteria and frozen sections have high specificity for ruling in failure [2]. As intraoperative synovial or tissue cultures are not available immediately, it could not provide convincing support at the time of re-implantation [4-6]. These limitations make intraoperative frozen section a better reference in judging infection at the time of re-implantation $[7,8]$.

Failed two-stage revisions discourage both patients and doctors. Under such circumstance, we desperately need to find out the reliable indicators and risk factors of these failed cases. We reviewed our recent two-stage total knee revision patients with positive culture results, and try to answer the following questions: (1) are intraoperative frozen sections, serum CRP, and ESR reliable references for the second-stage revision? (2) What are the risk factors for the failure of two-stage revision? (3) What is the proper timing for the second-stage revision?

\section{Methods}

Eighty-one periprosthetic knee infection patients from January 2010 to January 2014 with positive culture results were followed in this retrospective study. All patients received two-stage revision with articulated antibiotic-loaded cement spacer implantation. We have excluded patients with negative culture results, as they were hard to diagnose as PJI even with the MSIS criteria. Our cohort included 56 male and 25 female, the average age was $64.8 \pm 8.21$ (range 36-78) years. The mean follow-up time was $46.5 \pm 17.6$ (range 12-72) months after the second-stage surgeries. This study was approved by the institutional review board of our hospital, and all participants were informed and content.

All these patients showed suspicious symptoms of infection including periprosthetic fever, swelling or pain when they came to our clinic. Their medical histories were collected and laboratory tests including serum CRP and ESR were ran to help diagnose PJI. The threshold of CRP and ESR was $10 \mathrm{mg} / \mathrm{L}$ and $30 \mathrm{~mm} / \mathrm{h}$ according to
MSIS criteria, respectively. Synovial fluid aspirations and cultures were performed. The MSIS criteria were adopted to diagnose PJI. All these 81 patients were confirmed as PJI with the same phenotypic cultures. During the first stage, the former prosthesis was removed and a radical debridement was performed. At least three periprosthetic tissues were sent as culture samples. Another three periprosthetic tissues were collected as frozen section samples. Then, we mixed sensitive antibiotic into bone cement and made handcrafted spacers. The spacers were articulated in order to maintain soft tissue balancing. After the spacer was implanted, sensitive intravenous antibiotic treatment initiated. This treatment period lasted for at least 6 weeks. If the culture results suggested pathogens, such as fungal or mycobacterium tuberculosis, were hard to eradicate, the antibiotic treatment would extend. Patients were followed by phones after the spacer implantations until they finished the intravenous antibiotic treatment. The infection was considered as being eradicated with no clinical signs and symptoms, then they came back to our hospital for re-evaluation.

Before the second stage, serum CRP and ESR were tested again to reassess the control of infection and inflammation in the tissues. During the second-stage surgeries, at least three periprosthetic frozen sections and three tissue culture samples were collected after the removal of cement spacers. We employed the Feldman criterion for frozen section, which was interpreted as more than five neutrophils per high-power field $(\times 400)$ in at least five separate microscopic fields [9]. The following step depended on the overall consideration by surgeons, either implantation of a new prosthesis or another cement spacer.

These following situations were regarded as failure: (1) repeated spacer implantation; (2) recurrent infection with the same pathogen after new prosthesis implantations.

\section{Statistical analysis}

All data were entered into database and analyzed by SPSS software (Version 19.0. NY, USA). Quantitative data including age, height, weight, and BMI were described with mean and standard deviation (SD). The follow-up time, spacer detention time, and survival time were reported as median and range. Categorical data were described with percentages. We used Students' $t$ test to compare the difference of age, height, weight, BMI, and spacer detention time. Chi-square test was used to identify the difference of qualitative parameters. We also calculated the sensitivity and specificity of CRP, ESR, and Frozen section at time of second-stage revision. Receiver operating characteristic (ROC) curves were drawn to elevate the diagnostic accuracy of CRP, ESR, and frozen section at the second-stage revision. 
The area under the ROC curves (AUC) was also calculated. Cox proportional hazards model were adopted to explore the risk factors for failed two-stage surgeries. The $P$ values less than 0.05 were considered as statistically significant.

\section{Result}

\section{Diagnose of PJl and detected microorganisms}

The major clinical symptom of the cohort was persistent pain with impaired joint function. According to the pain grading of World Health Organization (WHO), 67 $(82.72 \%)$ patients were at grade II, while the rest 14 patients (17.28\%) were at grade III. Sinus tract was observed in 17 patients (21\%). Pre-operative joint aspiration culture identified pathogens in 52 patients before the first stage surgeries. And the other microorganisms were detected through the intra-operative tissue cultures. The most common pathogens were Staphylococcus, and $31.5 \%$ of them were methicillin-resistant. The atypical pathogens of PJI were detected in 27 patients. There were also nine patients infected with fungus including five Candida parapsilosis, three Candida albicans, and one aspergillus. Other microorganisms included Streptococcus and Escherichia coli (Table 1).

Table 1 Microorganisms detected in 81 periprosthetic knee infection patients

\begin{tabular}{llll}
\hline & Organism detected & N & Percentage \\
\hline Non methicillin-resistant & Staphylococcus aureus & 12 & $14.8 \%$ \\
& Staphylococcus & 10 & $12.3 \%$ \\
& epidermidis & & \\
& Coagulase-negative & 12 & $14.8 \%$ \\
& Staphylococcus & & \\
& Staphylococcus warneri & 3 & $3.7 \%$ \\
Methicillin-resistant & MRSE & 9 & $11.1 \%$ \\
Staphylococcus & MRSA & 8 & $9.88 \%$ \\
& Candida albicans & 3 & $3.7 \%$ \\
& Candida parapsilosis & 5 & $6.17 \%$ \\
& Aspergillus & 1 & $1.23 \%$ \\
Other pathogen & Streptococcus & 4 & $4.94 \%$ \\
& Escherichia coli & 5 & $6.17 \%$ \\
& $\begin{array}{l}\text { Pseudomonas } \\
\text { aeruginosa }\end{array}$ & 1 & $1.23 \%$ \\
& Enterococcus faecalis & 2 & $2.47 \%$ \\
& Mycobacterium & 2 & $2.47 \%$ \\
tuberculosis & & \\
NTM & 2 & $2.47 \%$ \\
& Klebsiella pneumoniae & 1 & $1.23 \%$ \\
& Acinetobacter & 1 & $1.23 \%$ \\
baumannii & & \\
\hline
\end{tabular}

MRSA methicillin-resistant Staphylococcus aureus, MRSE methicillin-resistant Staphylococcus epidermidis,

NTM non-tuberculosis mycobacterium

\section{Demographic information}

There were 71 patients (22 males and 49 females) received primary total knee arthroplasties for end-stage osteoarthritis, 5 ( 2 males and 3 females) for traumatic arthritis, and 5 (4 males and 1 female) for rheumatoid arthritis. The average age at primary TKA was $64.8 \pm 8.21$ years. The average height, weight, and BMI were $1.62 \pm 0.07 \mathrm{~m}, 69.5$ $\pm 11.6 \mathrm{~kg}$, and $26.31 \pm 3.6 \mathrm{~kg} / \mathrm{m}^{2}$, respectively. All these patients were followed after each stage surgery. The mean follow-up time after the second-stage revision was $46.5 \pm$ 17.6 months (range 12-72 months). The mean spacer detention time was $24.02 \pm 16.6$ weeks (range 12-96 week). The mean antibiotic treatment time was $7.59 \pm 2.54$ weeks (range 4-12 weeks).

\section{Unexpected repeated first-stage and failed second-stage revision}

The general information of patients with successful and failed two-stage revisions was compared with Students' $t$ test or chi-square test. No significant difference was found regarding age, height, weight, BMI, and pain severity. And there was also no difference of follow-up time (Table 2).

Seventy-one patients were successfully cured with two-stage protocol. The success rate of two-stage revision was $87.7 \%$. During our investigation, we observed five patients with failed first-stage surgeries and five with failed second stages. Five patients with failed first-stage revisions underwent repeated cement spacer implantation. And two of them were infected with non-tuberculosis mycobacterium, and they all received three spacer implantations before the final prostheses were implanted. The other five patients had reinfection with same pathogen after replacements of new prostheses. There were three patients that had sinus tract recurrence after second-stage revisions, two of them were infected with methicillin-resistant Staphylococcus, the other one was infected with Candida albicans (Table 3).

\section{Spacer detention time}

In our study, we discovered 16 week of spacer detention time is a valuable point for re-implantation. Forty patients received new prosthesis implantation with the spacer detention time between 12 and 16 weeks, and only three of them failed. The other 41 patients had new prostheses implanted with more than 16 weeks, and 7 of them endured failed two-stage revision. The difference was statistically significant (7.5 vs $17.1 \%, p=0.001)$.

\section{Reliable indicators at re-implantation}

We evaluated the diagnostic value of serum CRP, ESR, and intraoperative frozen section at the time of re-implantation. The AUCs proved both intraoperative frozen section was the most useful diagnostic indicator 
Table 2 Comparison between successful and failed two-stage patients

\begin{tabular}{llll}
\hline & $\begin{array}{l}\text { Successful } \\
\text { two-stage }\end{array}$ & $\begin{array}{l}\text { Failed } \\
\text { two-stage }\end{array}$ & $p$ value \\
\hline Number & 71 & 10 & $N$ \\
Age (years) & $65.14 \pm 8.45$ & $62.4 \pm 6.08$ & 0.326 \\
Height $(\mathrm{m})$ & $1.62 \pm 0.06$ & $1.65 \pm 0.09$ & 0.147 \\
Weight(kg) & $69.18 \pm 11.18$ & $72.10 \pm 14.76$ & 0.461 \\
BMI(kg/m $\left.{ }^{2}\right)$ & $26.30 \pm 3.51$ & $26.42 \pm 4.82$ & 0.919 \\
Pain severity & & & 0.171 \\
$\quad$ I & 42 & 3 & \\
$\quad$ II & 20 & 4 & \\
$\quad$ III & 9 & 3 & \\
Follow-up time (months) & $54.92 \pm 27.03$ & $43.0 \pm 25.30$ & 0.191 \\
Spacer detention time (weeks) & $23.97 \pm 17.28$ & $24.4 \pm 11.54$ & 0.94 \\
Antibiotic treatment time (weeks) & $7.23 \pm 2.14$ & $10.20 \pm 5.69$ & 0.135 \\
\hline
\end{tabular}

(FS: AUC $=0.84, p<0.05$, Fig. 1 ). While serum ESR was not available as diagnostic parameters at the time of re-implantation (AUC $=0.69, p=0.055)$.

At the time of re-implantation, intraoperative frozen section showed outstanding diagnostic capacity of both ruling out and ruling in infection with the sensitivity was 90\% (95\% confidence interval, 54.1-99.5\%) and specificity was $83.1 \%$ (95\% confidence interval, 71.9-90.6\%). The overall accuracy of FS was $84 \%$. Both serum CRP and ESR were useful in ruling in infection where the specificity was $88.7 \%$ (95\% confidence interval, $78.5-94.7 \%)$ and $84.5 \%(95 \%$ confidence interval $73.5-91.6 \%)$; while they had poor utility in ruling out infection with sensitivity that was only $40 \%$ (95\% confidence interval, $13.7-72.6 \%$ ) and $50 \%$ (95\% confidence interval, 20.1-80.0\%) (Table 4). The Youden Index of frozen section was 0.73 . This result indicated serum ESR and CRP were not good diagnostic indicators at the time of re-implantation with the Youden Index were 0.29 and 0.35 , respectively.

\section{Risk factors for failed two-stage TKA revision}

Our Cox proportional hazards model revealed that positive frozen section, atypical pathogen infection, and previous sinus tract were the risk factors for failed two-stage revisions (Table 5). If the patient had a sinus tract communicating to the joint, the failure rate for two-stage revision would increase 7.94 times (hazard rates[HR],7.94[1.701-37.093], $p=0.008$ ). At the time of re-implantation, if the frozen section shows more than five neutrophils per high-power field ( $\times 400$ magnification), the failure rate would increase 4.2 times (hazard rates [HR], 4.215 [1.047-16.959], $p=0.043)$. Patients infected atypical pathogen, the failure rate would increase 8.8 times (hazard rates [HR], 8.833[1.165-66.949], $p=0.035$ ). However, antibiotic treatment less than 6 weeks, positive serum CRP and ESR were not risk factors for failure.

Table 3 Information of failed second-stage revisions

\begin{tabular}{|c|c|c|c|c|c|c|c|c|}
\hline Number & Gender & Age & $\mathrm{BMI}$ & Diagnosis & Pathogen & $\begin{array}{l}\text { Spacer retention } \\
\text { period (weeks) }\end{array}$ & Failed details & Ending \\
\hline 4 & Male & 67 & 22.4 & $\mathrm{OA}$ & MRSE & 24 & $\begin{array}{l}\text { Sinus recurrence } 9 \text { months } \\
\text { after spacer implanted }\end{array}$ & $\begin{array}{l}\text { Three spacer implantations, one } \\
\mid \& D \text {, infection controlled. }\end{array}$ \\
\hline 16 & Male & 75 & 17.8 & $\mathrm{OA}$ & MRSA & 12 & $\begin{array}{l}\text { Sinus recurrence } 6 \text { months } \\
\text { after prosthesis implanted }\end{array}$ & $\begin{array}{l}\text { Two I\&D and insert exchange, } \\
\text { fusion }\end{array}$ \\
\hline 18 & Female & 65 & 27.7 & $\mathrm{OA}$ & Enterococcus faecalis & 12 & $\begin{array}{l}\text { Reinfection } 39 \text { months after } \\
\text { new prosthesis implanted }\end{array}$ & $\begin{array}{l}\text { Another one-stage revision, } \\
\text { infection controlled }\end{array}$ \\
\hline 23 & Female & 56 & 26.6 & OA & Group G Streptococcus & 24 & $\begin{array}{l}\text { Infection persist } 2 \text { months } \\
\text { after spacer implanted }\end{array}$ & $\begin{array}{l}\text { Two spacer implantation, } \\
\text { Two I\&D and insert exchange, } \\
\text { infection controlled }\end{array}$ \\
\hline 29 & Female & 63 & 23.9 & RA & Candida albicans & 48 & $\begin{array}{l}\text { Infection persist } 16 \text { months } \\
\text { after spacer implanted }\end{array}$ & $\begin{array}{l}\text { Two spacer implantation, } \\
\text { infection controlled }\end{array}$ \\
\hline 30 & Female & 59 & 28.9 & $\mathrm{OA}$ & MRSE & 20 & $\begin{array}{l}\text { Reinfection } 24 \text { months } \\
\text { after prosthesis implanted }\end{array}$ & $\begin{array}{l}\text { Another one-stage revision, } \\
\text { infection controlled }\end{array}$ \\
\hline 32 & Male & 64 & 22.5 & $\mathrm{OA}$ & NTM & 24 & $\begin{array}{l}\text { Infection persist } 9 \text { months } \\
\text { after spacer implanted }\end{array}$ & $\begin{array}{l}\text { Three spacer implantations, } \\
\text { infection controlled. }\end{array}$ \\
\hline 35 & Female & 58 & 31.3 & $\mathrm{OA}$ & Candida albicans & 40 & $\begin{array}{l}\text { Sinus recurrence } 6 \text { months } \\
\text { after prosthesis implanted }\end{array}$ & $\begin{array}{l}\text { One I\&D and insert exchange, } \\
\text { infection controlled }\end{array}$ \\
\hline 37 & Male & 63 & 34.3 & $\mathrm{OA}$ & NTM & 24 & $\begin{array}{l}\text { Infection persist } 10 \text { months } \\
\text { after spacer implanted }\end{array}$ & $\begin{array}{l}\text { Three time spacer implantations, } \\
\text { infection controlled }\end{array}$ \\
\hline 57 & Male & 54 & 28.7 & $\mathrm{OA}$ & Candida parapsilosis & 16 & $\begin{array}{l}\text { Reinfection } 4 \text { months } \\
\text { after prosthesis implanted }\end{array}$ & $\begin{array}{l}\text { One I\&D and insert exchange, } \\
\text { infection controlled }\end{array}$ \\
\hline
\end{tabular}




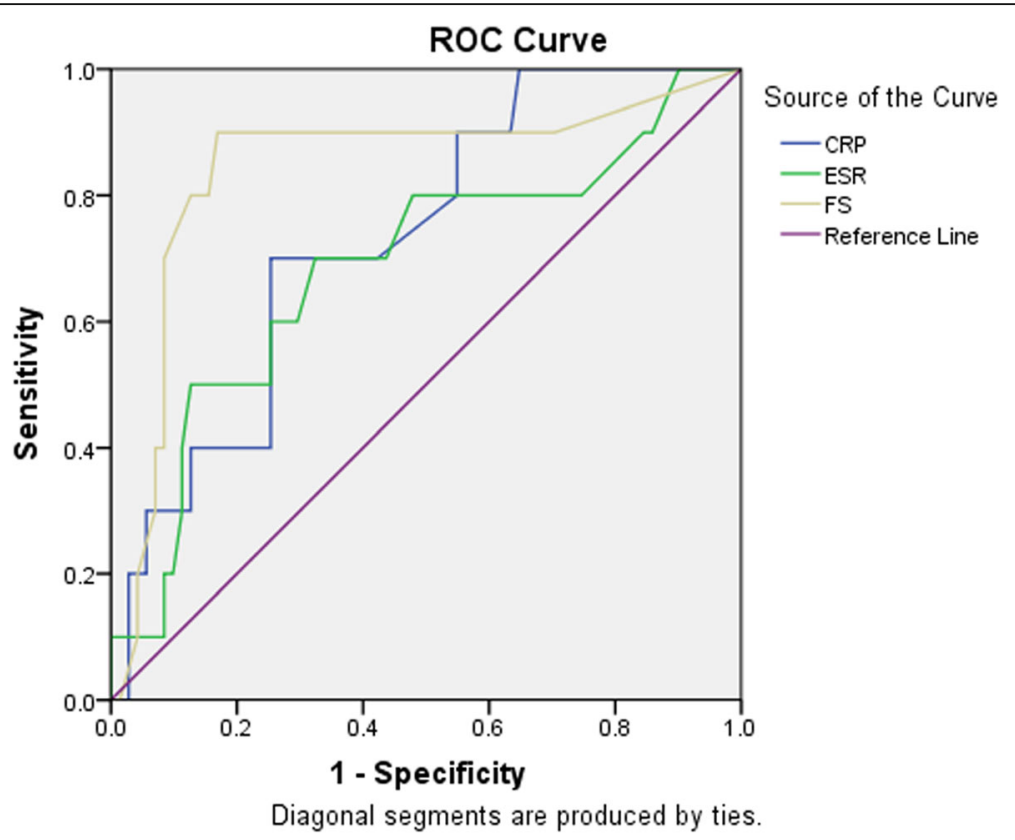

Fig. 1 ROC curve of intraoperative frozen section, serum CRP, and ES

\section{Discussion}

For a long period before the MSIS criteria, the diagnosis of PJI was quite challenging. Over the past several years, lots of researchers have proved the clinical value of MSIS criteria in diagnosing primary PJI [10]. Despite of many efforts on diagnosing of primary PJI, researchers seldom focused on the re-evaluation methods for the proper timing of re-implantation. As so far, there existed no consensus on this issue. However, the timing of re-implantation deserves more attention.

In our study, we have excluded suspicious PJI patients without pathogenic results, as these patients were hard to confirm as infection even with MSIS criteria [6, 11]. All selected 81 patients had positive culture results, PJI diagnoses were undisputed. The major microorganism detected was Staphylococcus, and 20\% of them were methicillin-resistant Staphylococcus. Our cohort also involved some rare pathogens, such as NTM and Candida albicans. At mean 46.5 months follow-up, the overall successful rate of two-stage revision in this study was $87.7 \%$, which was consistent with previous published researches in this field $[1,12]$. We observed 10 patients failed in the two-stage revision, among which there included three Candida and two NTM-infected patients. Our COX model indicated that atypical-infected TKA patients were 8.8 times more likely to fail a two-stage revision. All the 10 patients received multiple unexpected surgeries, which reminded us the high risk of failure when the pathogen was rare and needed longtime and combined antibiotic treatment.
To our acknowledgment, most surgeons judge the timing of re-implantation by clinical symptoms and certain laboratorial indicators. Our surgeons also followed this principle. But surgeons had to re-implant prostheses with risks under some circumstances, for example, patients were unable to afford or bear another spacer exchange. In our study, six patients underwent reimplantation with persistently elevated serum CRP and ESR, and eventually half of them failed.

The length of antibiotic treatment is very important for treating PJI. Bernard conducted a prospective nonrandomized study, and he found that 6 weeks of antibiotic treatment was sufficient for PJI [13]. Current studies suggested antibiotic treatment duration time should be held between 6 and 12 weeks [14]. In this study, the mean antibiotic treatment time between two stages was $7.11 \pm$ 2.07 weeks, and no difference was found between successful and failed patients. We found antibiotic treatment less than 6 weeks was not a relative risk factor for failed two-stage revision in our study $(p=0.542)$. We believe 6 weeks of antibiotic treatment was enough for treating infected TKA patients.

According to Ines, the optimal timing for re-implantation was 4 to 11 weeks after the first stage. In our study, the mean spacer detention time was 24 weeks for successful two-stage revision and 21 weeks for the 10 failed [15]. However, we discovered patients with spacer detention time between 12 and 16 weeks had lower failure rate. The least detention time in our study was 12 weeks, which made it impossible to analyze the actual effect of 
Table 4 Diagnostic evaluation of CRP, ESR and Frozen section at re-implantations

\begin{tabular}{lllllllr}
\hline CRP & $40 \%(13.7-72.6 \%)$ & $88.7 \%(78.5-94.7 \%)$ & $33.3 \%(11.3-64.6 \%)$ & $91.3 \%(81.3-96.4 \%)$ & $82.70 \%$ & 0.732 & 0.29 \\
ESR & $50 \%(20.1-80.0 \%)$ & $84.5 \%(73.5-91.6 \%)$ & $31.3 \%(12.1-58.5 \%)$ & $92.3 \%(82.2-97.1 \%)$ & $80.20 \%$ & 0.688 & 0.35 \\
FS & $90 \%(54.1-99.5 \%)$ & $83.1 \%(71.9-90.6 \%)$ & $42.3 \%(22.6-65.6 \%)$ & $98.3 \%(89.9-99.9 \%)$ & $84.00 \%$ & 0.843 & 0.73 \\
\hline
\end{tabular}

PPV positive predictive value, NPV negative predictive value, AUC area under the curve

any shorter detention time. Ines's study included culture negative patients, and the mean follow-up time was 20.5 months. We believe this discrepancy may need further investigation and follow-up.

Improvement of symptoms alone was not enough to determine the infectious status. Most surgeons made decisions on the decrement of inflammatory indicators. As joint aspiration samples may be difficult to obtain for some patients, especially for patients with sinus. Such situation made synovial white cell count and leukocyte strip difficult to complete for certain patient. Thus, serum CRP, ESR, and intraoperative frozen sections turned out to be more convenient indicators for the verdict of infection. At the time of re-implantation, our study showed that serum CRP and ESR had low sensitivity in ruling out infection. These two indicators showed poor diagnostic value in predicting failure of two-stage revision. It is speculated that persistent infection may produce limited efforts in laboratory parameters, and the cut-off values should be reevaluated.

In this study, the sensitivity and specificity of intraoperative FS is $90 \%$ and $83.1 \%$, and COX model revealed that positive frozen section is a high risk factors (HR 4.22, $p=0.043$ ), which makes us believe FS is an excellent parameter at the time of re-implantation. According to former meta-analysis, intraoperative FS was reported as a good indicator for predicting failure in culture-positive PJI patients [16]. Again, our result confirmed this conclusion through exclusion of culture negative patients. The sinus tract was found as a high risk factor for failure (HR 7.94, $p=0.008$ ). The sinus is usually generated by high virulence microorganisms, such as Staphylococcus aureus and Streptococcus. It means we should be more careful when treating PJI patients with sinus. The most common microorganism detected in PJI patients was Staphylococcus which usually account for nearly 40 $50 \%[14,17]$. Our study also presented the similar culture results with Staphylococcus infection rate $66.7 \%$. But we also detected $33.3 \%$ patients had atypical pathogen infection. Statistical analysis showed atypical pathogen PJI was a high risk factor for failed two-stage revision. Atypical pathogens of PJI patients are sometimes very difficult to incubate. Candida could grow on most medium, while NTM and fungal are high selective of growth environment, which requires special medium and prolonged culture time. Current literatures supported two-stage revision as the surgical management for these part patients [17-20].

Our study had some limitations. Firstly, this retrospective study only evaluated diagnostic efficiency of serum CRP, ESR, and FS. The negative culture result was excluded for a more authentic PJI cohort, whereas may lose some infected patients. More factors should be included to estimate inflammation of the patients. Secondly, the follow-up time is 46 months, which is relatively short for assessment of late reinfection and need further follow-up. Thirdly, the diet during the follow-up investigations is not taken into account. The specific diets which could prevent infections may be helpful for the surgical patients. Another deficiency is the low sample size; we only observed 10 failed two-stage revision patients, and it requires large sample to verify our results. And more prospective studies on timing of the re-implantation should be conducted.

\section{Conclusion}

The intraoperative frozen section is a good indicator at the time of re-implantation; the sensitivity and specificity is 90 and $83.1 \%$. Serum CRP and ESR showed poor diagnostic value at time of re-implantation. Atypical

Table 5 Risk factors for failed two-stage revision

\begin{tabular}{|c|c|c|c|c|c|c|c|c|}
\hline \multicolumn{9}{|l|}{ Variables in the equation } \\
\hline & \multirow[t]{2}{*}{ B } & \multirow[t]{2}{*}{ SE } & \multirow[t]{2}{*}{ Wald } & \multirow[t]{2}{*}{$d f$} & \multirow[t]{2}{*}{ Sig. } & \multirow[t]{2}{*}{$\operatorname{Exp}(B)$} & \multicolumn{2}{|c|}{$95.0 \% \mathrm{Cl}$ for $\operatorname{Exp}(\mathrm{B})$} \\
\hline & & & & & & & Lower & Upper \\
\hline Sinus & 2.072 & 0.786 & 6.944 & 1 & 0.008 & 7.942 & 1.701 & 37.093 \\
\hline Antibiotic treatment less than 6 weeks & -0.596 & 0.978 & 0.372 & 1 & 0.542 & 0.551 & 0.081 & 3.746 \\
\hline Positive CRP & 0.313 & 0.835 & 0.141 & 1 & 0.708 & 1.368 & 0.266 & 7.029 \\
\hline Positive ESR & 1.276 & 0.787 & 2.629 & 1 & 0.105 & 3.582 & 0.766 & 16.753 \\
\hline Positive FS & 1.439 & 0.710 & 4.102 & 1 & 0.043 & 4.215 & 1.047 & 16.959 \\
\hline Atypical pathogen infection & 2.178 & 1.033 & 4.444 & 1 & 0.035 & 8.833 & 1.165 & 66.949 \\
\hline
\end{tabular}


pathogen infection, positive FS, and previous sinus were high risk factors for failure of two-stage revision. The antibiotic treatment less than 6 weeks would not increase the failure rate. Spacer detention time between 12 and 16 weeks had higher success rate than over 16 weeks. The proper timing of re-implantation should be combined with disappearance of clinical symptoms and negative intraoperative FS with spacer detention time at 12 to 16 weeks.

\section{Abbreviations}

AUC: Area under the ROC curves; CRP: C-reaction protein; ESR: Erythrocyte sedimentation; MSIS: Musculoskeletal Infection Society; PJI: Periprosthetic joint infection; ROC: Receiver operating characteristic; SD: Standard deviation

\section{Availability of data and materials}

The data supporting our findings can be found in PubMed.

\section{Authors' contributions}

$\mathrm{JF}$ and $\mathrm{JC}$ conceived and designed the experiments. $\mathrm{MN}, \mathrm{HL}$, and $\mathrm{XL}$ conceived and performed the experiments. WC and $Y Z$ prepared the figures. $W C, Y Z$, and LH wrote the main manuscript text. All authors reviewed the manuscript. All authors read and approved the final manuscript.

\section{Ethics approval and consent to participate}

The following methods were carried out in accordance with the approved guidelines. All experimental protocols were approved by Chinese People's Liberation Army General Hospital (301 Hospital) Committee, written informed consent was obtaining from every patient.

\section{Consent for publication}

We obtained permission from the participants to publish their data.

\section{Competing interests}

The authors declare that they have no competing interests.

\section{Publisher's Note}

Springer Nature remains neutral with regard to jurisdictional claims in published maps and institutional affiliations.

\section{Author details}

'Department of Orthopaedics, Chinese People's Liberation Army General Hospital (301 Hospital), Beijing 100853, China. ${ }^{2}$ Department of Orthopaedics, Beijing Jishuitan Hospital, Beijing, China.

Received: 13 March 2018 Accepted: 6 July 2018

Published online: 29 August 2018

\section{References}

1. Masters JP, Smith NA, Foguet P, Reed M, Parsons H, Sprowson AP. A systematic review of the evidence for single stage and two stage revision of infected knee replacement. BMC Musculoskelet Disord. 2013;14:222.

2. George J, Kwiecien G, Klika AK, Ramanathan D, Bauer TW, Barsoum WK, Higuera CA. Are frozen sections and MSIS criteria reliable at the time of reimplantation of two-stage revision arthroplasty? Clin Orthop Relat Res. 2016:474(7):1619.

3. Ghanem E, Azzam K, Seeley M, Joshi A, Parvizi J. Staged revision for knee arthroplasty infection: what is the role of serologic tests before reimplantation? Clin Orthop Relat Res. 2009;467(7):1699.

4. McGrory BJ, Becker MW. Screening for infection to determine treatment in second-stage reimplantation of total knee prostheses. J Bone Joint Surg Am. 2001;83-a(9):1433.

5. Mont MA, Waldman BJ, Hungerford DS. Evaluation of preoperative cultures before second-stage reimplantation of a total knee prosthesis complicated by infection. A comparison-group study. J Bone Joint Surg Am. 2000;82a(11):1552

6. Huang RHC. Culture-negative periprosthetic joint infection does not preclude infection control. Clin Orthop Relat Res. 2012;470(10):2717.
7. Della Valle CJ, Bogner E, Desai P, Lonner JH, Adler E, Zuckerman JD, Di Cesare PE. Analysis of frozen sections of intraoperative specimens obtained at the time of reoperation after hip or knee resection arthroplasty for the treatment of infection. J Bone Joint Surg Am. 1999;81(5):684.

8. Bori GSA. Neutrophils in frozen section and type of microorganism isolated at the time of resection arthroplasty for the treatment of infection. Arch Orthop Trauma Surg. 2009;129(5):591.

9. Feldman DS, Lonner JH, Desai P, Zuckerman JD. The role of intraoperative frozen sections in revision total joint arthroplasty. J Bone Joint Surg Am. 1807;77(12):1995.

10. Parvizi J, Gehrke T, Chen AF. Proceedings of the international consensus on Periprosthetic joint infection. Bone Joint J. 2013;95-b(11):1450.

11. Berbari EFMC. Culture-negative prosthetic joint infection. Clin Infect Dis. 2007;24:1113.

12. Kunutsor SK, Whitehouse MR, Lenguerrand E, Blom AW, Beswick AD. Reinfection outcomes following one- and two-stage surgical revision of infected knee prosthesis: a systematic review and meta-analysis. PLoS One. 2016;11(3):e0151537.

13. Bernard L, Legout L, Zurcher-Pfund L, Stern R, Rohner P, Peter R, Assal M, Lew $D$, Hoffmeyer $P$, Uckay I. Six weeks of antibiotic treatment is sufficient following surgery for septic arthroplasty. J Infect. 2010;61(2):125.

14. Douglas R, Osmon EFB. Diagnosis and Management of Prosthetic Joint Infection: clinical practice guidelines by the Infectious Diseases Society of America. Clin Infect Dis. 2013;56(1):e1.

15. Vielgut I, Sadoghi P, Wolf M, Holzer L, Leithner A, Schwantzer G, Poolman R, Frankl B, Glehr M. Two-stage revision of prosthetic hip joint infections using antibiotic-loaded cement spacers: when is the best time to perform the second stage? Int Orthop. 2015;39(9):1731.

16. Tsaras G, Maduka-Ezeh A. Utility of intraoperative frozen section histopathology in the diagnosis of periprosthetic joint infection: a systematic review and metaanalysis. J Bone Joint Surg Am. 2012;94(18):1700.

17. Peel TN, Cheng AC. Microbiological aetiology, epidemiology, and clinical profile of prosthetic joint infections: are current antibiotic prophylaxis guidelines effective? Antimicrob Agents Chemother. 2012;56(5):2386.

18. Azzam K, Parvizi J, Jungkind D, Hanssen A, Fehring T, Springer B, Bozic K, Della Valle C, Pulido L, Barrack R. Microbiological, clinical, and surgical features of fungal prosthetic joint infections: a multi-institutional experience. J Bone Joint Surg Am. 2009;91(Suppl 6):142.

19. Cobo F, Rodriguez-Granger J, Lopez EM, Jimenez G, Sampedro A, Aliaga-Martinez L, Navarro-Mari JM. Candida-induced prosthetic joint infection. A literature review including 72 cases and a case report. Infectious Dis (Lond). 2017:49(2):81.

20. Dutronc H, Dauchy FA, Cazanave C, Rougie C, Lafarie-Castet S, Couprie B, Fabre T, Dupon M. Candida prosthetic infections: case series and literature review. Scand J Infect Dis. 2010;42(11-12):890.

Ready to submit your research? Choose BMC and benefit from:

- fast, convenient online submission

- thorough peer review by experienced researchers in your field

- rapid publication on acceptance

- support for research data, including large and complex data types

- gold Open Access which fosters wider collaboration and increased citations

- maximum visibility for your research: over $100 \mathrm{M}$ website views per year

At $\mathrm{BMC}$, research is always in progress.

Learn more biomedcentral.com/submission 